\section{Qualidade de vida dos indivíduos expostos ao césio-137, em Goiânia, Goiás, Brasil}

\author{
Quality of life in individuals exposed to \\ cesium-137 in Goiânia, Goiás State, Brazil
}

\author{
Calidad de vida de las personas expuestas al \\ cesio-137, en Goiânia, Goiás, Brasil
}

\author{
Silvana Cruz Fuini 1 \\ Rafael Souto 2 \\ Geraldo Francisco do Amaral 3 \\ Rita Goreti Amaral 4
}

\author{
1 Programa de Pós-graduação \\ Ciências da Saúde, \\ Universidade Federal de \\ Goiás, Goiânia, Brasil. \\ 2 Instituto de Patologia \\ Tropical e Saúde Pública, \\ Universidade Federal de \\ Goiás, Goiânia, Brasil. \\ ${ }^{3}$ Faculdade de Medicina, \\ Universidade Federal de \\ Goiás, Goiânia, Brasil. \\ 4 Faculdade de Farmácia, \\ Universidade Federal de \\ Goiás, Goiânia, Brasil. \\ Correspondência \\ S. C. Fuini \\ Programa de Pós-graduação \\ Ciências da Saúde, \\ Universidade Federal de Goiás. \\ Rua 17-A, 1419, Setor \\ Aeroporto, Goiânia, GO \\ 74075-160, Brasil. \\ silvana.fuini@gmail.com
}

\section{Abstract}

This cross-sectional observational study in Goiânia, Goiás State, Brazil in a sample of 56\% of 111 individuals exposed to cesium-137 aimed to evaluate their quality of life and associations with socio-demographic factors. The study used the WHOQOL-BREF and the Monitoring System for Radiation Victims. Participants were divided according to international criteria: Group I - radiation dermatitis and / or cytogenetic dosimetry above 20 rads $(n=33)$ and Group II - cytogenetic dosimetry $\leq 20$ rads $(n=29)$, totaling 62 subjects. Among the WHOQOL-BREF domains, environment showed the highest mean scores (59.88, $S D=20.39)$ and psychological the lowest (53.02, $S D=17.98)$. Associations between the physical, psychological, and social domains were significant for the age variable. There was no difference between groups. The association between sociodemographic factors and quality of life was not significant. Radiation victims suffer considerable impact on quality of life, with persistent psychosocial problems, especially among those older than 41 years.

Quality of Life; Cesium; Psychosocial Impact; Accidents

\section{Resumo}

Estudo observacional transversal realizado em Goiânia, Goiás, Brasil, em uma amostra de 56\% de 111 pessoas, objetivando avaliar a qualidade de vida dos indivíduos expostos ao césio-137 e sua associação com fatores sociodemográficos. Utilizou-se o WHOQOL-BREF e o Sistema de Monitoramento dos Radioacidentados. Os participantes foram divididos segundo critérios internacionais em: Grupo I - radiodermites el ou dosimetria citogenética acima de 20 rads ( $n=33) ;$ e Grupo II - dosimetria citogenética $\leq 20$ rads $(n=29)$, totalizando 62 sujeitos. Dentre os domínios do WHOQOL-BREF, o meio ambiente apresentou a média de escores mais alta $(59,88$; $D P=20,39)$ e o psicológico a média mais baixa $(53,02 ; D P=17,98)$. As associações entre os domínios físico, psicológico e relações sociais foram significativas para a variável idade. Não houve diferença entre grupos. A associação entre os fatores sociodemográficos e qualidade de vida não foi significativa. Os radioacidentados sofrem considerável impacto na qualidade de vida, com persistência de problemas psicossociais, especialmente para aqueles com mais de 41 anos.

Qualidade de Vida; Césio; Impacto Psicossocial; Acidentes 


\section{Introdução}

O acidente radioativo ocorrido em setembro de 1987, na cidade de Goiânia, Estado de Goiás, Brasil, trouxe problemas sérios que perduram até os dias de hoje 1. Esse evento foi provocado por meio da ruptura de um aparelho radioterápico abandonado em uma clínica médica desativada, e posteriormente agravado pelo manuseio incorreto da cápsula que continha o césio-137 (isótopo radioativo) por pessoas leigas no assunto 2 .

Na época, em torno de 112 mil pessoas foram envolvidas no acidente, cerca de uma centena diretamente e outras centenas indiretamente incluindo familiares, vizinhos e agentes públi$\cos 2,3$. Desde então é realizado regularmente por uma unidade de saúde específica, atualmente denominada Centro de Assistência aos Radioacidentados (C.A.R.A.) ${ }^{3,4}$, que faz parte da estrutura da Secretaria de Saúde de Goiás, o monitoramento das pessoas envolvidas. Até os dias de hoje, os resultados do monitoramento não apontam dados estatisticamente significantes para morbimortalidade associada aos efeitos da radiação ionizante. Entretanto, pesquisadores recomendam o prosseguimento dos estudos, tendo em vista os efeitos tardios decorrentes do acidente radioativo 1,5 .

Dessa forma, os diversos aspectos relacionados à saúde e fatores psicossociais devem ser investigados nas populações potencialmente expostas à radioatividade, sobretudo, tomandose em conta o trauma, o medo generalizado e o estresse inerentes aos efeitos desse tipo de evento 6 . Estudos apontam que aproximadamente $75 \%$ dos indivíduos expostos a contaminações por acidentes nucleares apresentam alguma forma de sintomas psicológicos, desde a incapacidade de dormir à dificuldade de concentração e isolamento social. Entre aqueles com maior risco de efeitos significativos encontram-se as crianças, mulheres (grávidas e mães de crianças pequenas), idosos e pessoas com um histórico de transtorno psiquiátrico 7,8. Além disso, os indivíduos expostos têm uma alta taxa de transtorno do estresse pós-traumático (TEPT), o que colabora para a percepção de que alguns aspectos relacionados à qualidade de vida estão afetados 9 .

Nesse contexto, a qualidade de vida é entendida a partir de novos paradigmas que têm influenciado as práticas do setor saúde, levando-se em conta que o processo saúde/doença é complexo e abrange aspectos econômicos, socioculturais, experiência pessoal e estilos de vida. De acordo com esses novos aspectos, a melhoria da qualidade de vida passa a ser um dos resultados esperados pelas práticas assistenciais promovidas por todas as iniciativas de saúde que movem esforços nesta direção ${ }^{10,11}$. Sendo assim, a avaliação da qualidade de vida do paciente é reconhecida como uma importante área do conhecimento científico, em razão do seu conceito se interpor ao de saúde. Tal avaliação tem a vantagem de incluir aspectos subjetivos geralmente não abordados por outros critérios de avaliação 12 .

O interesse pelo conhecimento da qualidade de vida dos radioacidentados configura-se como uma preocupação constante da população do Estado de Goiás e especialmente do corpo clínico do C.A.R.A., sobretudo, devido à recorrência no atendimento psicossocial e à busca frequente pelos serviços médicos e por respostas às dúvidas que ainda persistem.

$\mathrm{O}$ referido fato corrobora com estudos que consideram o impacto na saúde mental como o maior problema de saúde pública desencadeado por acidentes dessa natureza e que, muitas vezes, esses transtornos vêm acompanhado de um grande número de queixas somáticas inespecíficas 8,9,13,14. Nesse aspecto, é reconhecido pelo corpo clínico do C.A.R.A. que desde a ocorrência do acidente radioativo com o césio-137 foi dada ênfase aos aspectos físicos das vítimas do acidente, em detrimento ao gerenciamento dos problemas sociais e psicológicos 15 .

Assim, este trabalho teve como objetivo avaliar a qualidade de vida dos indivíduos expostos ao césio-137, durante acidente ocorrido em Goiânia, e sua associação com fatores sociodemográficos.

\section{Métodos}

Trata-se de um estudo observacional transversal realizado no período de janeiro a agosto de 2011, cuja população-alvo foi composta por indivíduos expostos à radiação pelo césio-137, na cidade de Goiânia, devidamente cadastrados no C.A.R.A., unidade da Secretaria de Estado da Saúde de Goiás.

Desde a época do acidente radioativo, os pacientes cadastrados no C.A.R.A. foram categorizados por grupos (I, II e III) de acordo com as normas da International Atomic Energy Agency (IAEA), levando-se em conta critérios de classificação conforme a gravidade das lesões cutâneas e da intensidade de contaminação interna e externa. O Grupo I é composto por indivíduos com radiodermites e/ou dosimetria citogenética acima de 20rads. O II é formado por indivíduos com dosimetria citogenética $\leq 20$ rads. O Grupo III é constituído por agentes públicos, voluntários, familiares das vítimas e vizinhos 2,3 .

Foram incluídos os sujeitos dos Grupos I e II com mais de 18 anos. Excluiu-se os indivíduos 
com prejuízo cognitivo e os integrantes do Grupo III, em razão de que a maioria destes não foi monitorada na época do acidente, portanto há poucos registros da dosimetria correspondente ao nível de exposição ao césio-137 3 . Ainda, o Grupo III encontra-se em processo de cadastramento no C.A.R.A., na medida em que consegue o reconhecimento (seja administrativo ou judicial) de seu direito de receber os benefícios (pensões) concedidos por força de lei 3 .

Inicialmente, os indivíduos dos Grupos I e II foram convidados por carta-convite e contatos telefônicos para participarem do estudo. Do total de 111 sujeitos que atendiam aos critérios de inclusão, apenas 62 aceitaram participar da pesquisa (Grupo I $n=33$; Grupo II $n=29$ ). Após essa etapa, o questionário foi autoaplicado sob a orientação da pesquisadora responsável, em encontros realizados nas residências dos sujeitos ou nas dependências do C.A.R.A.

O instrumento de coleta de dados para avaliar a qualidade de vida foi o questionário World Health Organization Quality of Life (WHOQOLBREF), desenvolvido pela Organização Mundial da Saúde (OMS) e validado no Brasil por Fleck et al. 10. Contém 26 perguntas, sendo duas questões gerais de qualidade de vida - a primeira refere-se à autopercepção da qualidade de vida e a outra sobre a satisfação com a saúde. As demais 24 questões são distribuídas em quatro domínios: físico, psicológico, relações sociais e meio ambiente. Cada domínio é representado por facetas e suas perguntas foram formuladas para uma escala de respostas do tipo Likert (1 a 5), que é um tipo de escala de resposta psicométrica usada habitualmente em questionários, mais utilizada em pesquisas de opinião. Nessa escala é solicitado aos entrevistados que indiquem o seu grau de concordância ou discordância com uma informação que está sendo medida. Atribui-se valores numéricos e/ou sinais às respostas para refletir a força e a direção da reação do entrevistado à declaração. As declarações de concordância devem receber valores positivos ou altos, e as declarações das quais discordam devem receber valores negativos ou baixos. A pontuação total da atitude de cada respondente é dada pela somatória das pontuações obtidas para cada afirmação 16,17 .

O valor mínimo dos escores de cada domínio do WHOQOL-BREF é zero e o máximo é 100. O escore de cada domínio é obtido em uma escala positiva, isto é, quanto mais alto o escore, melhor a qualidade de vida naquele domínio 11,18.

Para obtenção das informações sociodemográficas utilizou-se o banco de dados do Sistema de Monitoramento dos Radioacidentados (SISRAD) disponível no C.A.R.A. O SISRAD é um software desenvolvido para informatizar e organizar o monitoramento e acompanhamento das vítimas do acidente ${ }^{3}$. As variáveis analisadas foram idade, sexo, grau de escolaridade e religião. Para a análise tomou-se por base duas faixas etárias (18 a 40 anos e 41 ou mais), de acordo com o protocolo clínico/laboratorial de monitoramento desses indivíduos, definido pela IAEA, que divide os mesmos nestas duas faixas etárias para a realização dos procedimentos estabelecidos.

A análise estatística foi realizada pelo programa SPSS for Windows, versão 15.0 (SPSS Inc., Chicago, Estados Unidos). Foi empregada a sintaxe WHOQOL-BREF no SPSS para avaliação dos escores do instrumento de qualidade de vida e posteriores correlações, baseando-se no coeficiente de Pearson. Para a análise da comparação das variáveis sociodemográficas e os domínios do WHOQOL-BREF foi utilizado o teste $t$ de Student e Anova para os dados normais. Para verificar a frequência das variáveis sexo e idade com os domínios do WHOQOL-BREF foi utilizado o teste qui-quadrado. Considerou-se como nível de significância o valor de $5 \%(p<0,05)$.

Este trabalho foi aprovado pelo Comitê de Ética em Pesquisa Dr. Henrique Santillo da Secretaria de Estado da Saúde de Goiás, sob protocolo no 0016.0.177.000-10.

\section{Resultados}

A população analisada foi composta por 62 sujeitos, correspondendo a $56 \%$ do universo de radioacidentados cadastrados no C.A.R.A, distribuídos por Grupos I e II. Do total de 111 indivíduos, 49 se negaram a participar da pesquisa, como pode ser observado na Tabela 1. Verificou-se que na comparação da distribuição de todas as variáveis analisadas no grupo não investigado houve diferença significativa na variável religião $(\mathrm{p}=0,001)$ e no grupo investigado na variável escolaridade $(0,047)$.

Os resultados sobre a percepção da qualidade de vida relacionada às duas questões genéricas do WHOQOL-BREF apresentaram na primeira questão - em que os indivíduos avaliaram a própria qualidade de vida - 44,9\% como "boa"; $6,5 \%$ "muito boa"; e 9,5\% "ruim" e "muito ruim". Desses, 39,9\% responderam como "nem ruim, nem boa”. Quanto à segunda questão, que mensura a satisfação dos sujeitos em relação à própria saúde, $35,4 \%$ disseram estar "satisfeitos"; $11,4 \%$ "muito satisfeitos"; e 37,7\% consideravam-se "insatisfeitos" e "muito insatisfeitos". O restante, $16,4 \%$, disse estar "nem satisfeito, nem insatisfeito". Ao separar os grupos, observou-se na média geral de cada questão que tanto o Grupo I como o 
Distribuição das variáveis de acordo com cada aspecto social por Grupos (I e II) na população de radioacidentados, nos grupos não investigado e investigado. Goiânia, Goiás, Brasil, 2011.

\begin{tabular}{|c|c|c|c|c|c|c|c|c|c|c|}
\hline \multirow[t]{3}{*}{ Aspecto social } & \multicolumn{5}{|c|}{ Grupo não investigado } & \multicolumn{5}{|c|}{ Grupo investigado } \\
\hline & \multicolumn{2}{|c|}{ Grupo I $(n=29)$} & \multicolumn{2}{|c|}{ Grupo II $(n=20)$} & \multirow{2}{*}{$\begin{array}{l}\text { Valor } \\
\text { de } p\end{array}$} & \multicolumn{2}{|c|}{ Grupo I $(n=33)$} & \multicolumn{2}{|c|}{ Grupo II $(n=29)$} & \multirow{2}{*}{$\begin{array}{l}\text { Valor } \\
\text { de } p\end{array}$} \\
\hline & $\mathrm{n}$ & $\%$ & $\mathrm{n}$ & $\%$ & & $\mathrm{n}$ & $\%$ & $\mathrm{n}$ & $\%$ & \\
\hline \multicolumn{11}{|l|}{ Sexo } \\
\hline Masculino & 18 & 62,1 & 9 & 45,0 & & 18 & 54,5 & 14 & 48,3 & \\
\hline Feminino & 11 & 37,9 & 11 & 55,0 & 0,187 & 15 & 45,5 & 15 & 51,7 & 0,799 * \\
\hline \multicolumn{11}{|l|}{ Idade (anos) } \\
\hline $18|-| 40$ & 11 & 37,9 & 13 & 65,0 & & 14 & 42,4 & 17 & 58,6 & \\
\hline$>41$ & 18 & 62,1 & 7 & 35,0 & 0,058 & 19 & 57,6 & 12 & 46,4 & 0,309 ** \\
\hline \multicolumn{11}{|l|}{ Escolaridade } \\
\hline Ensino Fundamental & 17 & 58,6 & 7 & 35,0 & & 9 & 36,0 & 15 & 51,7 & \\
\hline Ensino Médio & 8 & 27,6 & 12 & 60,0 & & 11 & 44,0 & 12 & 41,4 & \\
\hline Ensino Superior & 4 & 13,8 & 1 & 5,0 & 0,071 & 5 & 20,0 & 2 & 6,9 & 0,047 * \\
\hline \multicolumn{11}{|l|}{ Religião } \\
\hline Não possui & 3 & 10,3 & 1 & 5,0 & & 6 & 18,2 & 2 & 6,9 & \\
\hline Católico & 5 & 17,2 & 6 & 30,0 & & 18 & 54,5 & 19 & 65,5 & \\
\hline Espírita & 6 & 20,7 & 13 & 65,0 & & 1 & 3,0 & 1 & 3,4 & \\
\hline Evangélico & 15 & 51,7 & 0 & 0,0 & 0,001 & 8 & 24,2 & 7 & 24,1 & 0,606 * \\
\hline
\end{tabular}

* Teste qui-quadrado;

** Teste exato de Fisher.

II apresentaram os maiores escores na primeira e os menores na segunda questão, como se segue: Grupo I com 61,36 e 50,00 e o Grupo II com 62,93 e 56,90, respectivamente.

Dentre o universo de indivíduos que avaliaram sua qualidade de vida de modo geral como boa/muito boa e ruim/muito ruim, considerando a média nos domínios maior que 70 e menor que 30, destaca-se que no Grupo I cerca de 50\% eram homens e $50 \%$ mulheres, também apresentaram o mesmo porcentual nas duas faixas etárias (18-40 anos e 41 ou mais). Não houve predominância de algum grau de escolaridade e religião. Já no Grupo II a maioria era de homens e com idades entre 18 a 40 anos. Também, as variáveis escolaridade e religião encontram-se pulverizadas. Nesses dois grupos, as menores médias $(<30)$ concentraram-se nos domínios físico e psicológico e as maiores (> 70) nos domínios relações sociais e meio ambiente, com predominância neste último.

A Tabela 2 mostra os valores da distribuição dos índices de qualidade de vida em cada domínio do WHOQOL-BREF, indicando que não houve diferença. Para o Grupo I, o domínio meio ambiente apresentou a maior média de escores, enquanto que a menor média foi para o domínio relações sociais. Quanto ao Grupo II, o domínio meio ambiente indicou a média de escores mais alta e o domínio psicológico a menor pontuação. Quando analisamos os dois grupos de forma geral, verificou-se que o domínio psicológico obteve a menor média e o domínio meio ambiente a maior média.

A Tabela 3 mostra a comparação dos dados gerais separados por duas faixas etárias de 18 a 40 anos e com mais de 41 . Os resultados apresentaram diferença para a variável idade referente aos domínios físico, psicológico e relações sociais $(\mathrm{p}<0,05)$. Somente o domínio meio ambiente não foi estatisticamente significativo $(p>0,05)$.

A Tabela 3 mostra, ainda, que não houve diferença $(p>0,05)$ na variável sexo entre os domínios do WHOQOL-BREF. Apesar de não haver diferença, as mulheres apresentaram escores mais baixos em comparação aos homens em todos os domínios.

Ao analisar a variável idade separada por grupo, foi verificada diferença no Grupo I nas duas faixas etárias, referentes aos domínios psicológico $(p=0,017)$ e relações sociais $(p=0,004)$. Quanto à variável sexo separada por grupo, constatouse que não foi significante em todos os domínios, cujos escores ficaram assim representados nos Grupos I e II, respectivamente: domínio físico 52,86/53,81; domínio psicológico 50,56/53,06; 
Média e Desvio Padrão (DP) dos domínios do WHOQOL-BREF de acordo com o Grupo (I e II) e no geral, na amostra de indivíduos expostos ao césio-137. Goiânia, Goiás, Brasil, 2011.

\begin{tabular}{|c|c|c|c|c|c|c|}
\hline Domínios WHOQOL-BREF & $\mathbf{n}$ & Média & DP & Mínimo * & Máximo ** & Valor de $p$ \\
\hline \multicolumn{7}{|l|}{ Físico } \\
\hline Grupo I & 33 & 53,68 & 16,06 & 17,86 & 82,14 & \\
\hline Grupo II & 29 & 55,91 & 17,45 & 25,00 & 92,86 & \\
\hline Total & 62 & 54,72 & 16,62 & 17,86 & 92,86 & 0,602 \\
\hline \multicolumn{7}{|l|}{ Psicológico } \\
\hline Grupo I & 33 & 50,88 & 17,61 & 12,50 & 87,50 & \\
\hline Grupo II & 29 & 55,46 & 18,40 & 25,00 & 87,50 & \\
\hline Total & 62 & 53,02 & 17,98 & 12,50 & 87,50 & 0,322 \\
\hline \multicolumn{7}{|l|}{ Relações sociais } \\
\hline Grupo I & 33 & 49,75 & 21,60 & 0,00 & 83,33 & \\
\hline Grupo II & 29 & 58,05 & 17,74 & 16,67 & 83,33 & \\
\hline Total & 62 & 53,63 & 20,17 & 0,00 & 83,33 & 0,106 \\
\hline \multicolumn{7}{|l|}{ Meio ambiente } \\
\hline Grupo I & 33 & 61,27 & 19,75 & 25,00 & 93,75 & \\
\hline Grupo II & 29 & 58,30 & 21,33 & 15,63 & 100,00 & \\
\hline Total & 62 & 59,88 & 20,39 & 15,63 & 100,00 & 0,571 \\
\hline
\end{tabular}

Teste t de Student.

* Mínimo: 0;

** Máximo: 100

domínio relações sociais 46,67/55,56; domínio meio ambiente 59,79/51,88).

A Tabela 4 mostra a correlação entre os domínios do WHOQOL-BREF. Verifica-se que todos os domínios foram significativos e apresentaram relação moderada. Os maiores coeficientes foram encontrados nos domínios psicológico e físico. $\mathrm{O}$ domínio das relações sociais apresentou os coeficientes de correlação mais baixos.

\section{Discussão}

Os resultados apresentados pelos indivíduos expostos ao césio-137 no questionário WHOQOLBREF, considerando uma escala de 0 a 100, apresentaram, de forma crescente, para os domínios psicológico, relações sociais, físico e meio ambiente valores entre 53,02 e 59,88. Sendo que, as pessoas com mais de 41 anos, mulheres e os sujeitos do Grupo I, indicaram menores escores, principalmente quanto à avaliação geral da própria saúde e nos domínios psicológico e relações sociais.
Esses resultados são consistentes com os apresentados por Miranda et al. 19 em um estudo constituído por um follow up da população de radioacidentados de Goiânia em dois períodos (3 e 15 anos após o evento), cujo objetivo foi analisar a percepção das pessoas diretamente atingidas sobre os efeitos do acidente. Os resultados apontaram que a percepção de problemas psicológicos e de saúde geral permaneceu elevada nos dois períodos estudados, tanto para os indivíduos do Grupo I como para os do Grupo II.

Os resultados encontrados no presente trabalho são semelhantes aos verificados na população envolvida no acidente de Chernobyl, Ucrânia. Havenaar et al. 9, em um levantamento epidemiológico realizado após o desastre de Chernobyl, revelaram que os envolvidos tiveram substancialmente maiores taxas de queixas de saúde e sofrimento psíquico do que os habitantes de uma região não afetada. Além disso, Rahu 20 ressaltou que o desastre de Chernobyl teve um impacto de longo prazo sobre o bemestar físico e psicológico, doenças e na qualidade de vida das pessoas envolvidas. 
Comparação dos domínios do WHOQOL-BREF em relação ao sexo e faixa etária na amostra de indivíduos expostos ao césio-137. Goiânia, Goiás, Brasil, 2011.

\begin{tabular}{|c|c|c|c|}
\hline Domínios WHOQOL-BREF & $\mathrm{n}$ & Média & Valor de $p$ \\
\hline \multicolumn{4}{|l|}{ Sexo } \\
\hline \multicolumn{4}{|l|}{ Físico } \\
\hline Masculino & 32 & 56,03 & \\
\hline Feminino & 30 & 53,33 & \\
\hline Total & 62 & 54,72 & 0,528 \\
\hline \multicolumn{4}{|l|}{ Psicológico } \\
\hline Masculino & 32 & 54,17 & \\
\hline Feminino & 30 & 51,81 & \\
\hline Total & 62 & 53,02 & 0,610 \\
\hline \multicolumn{4}{|l|}{ Relações sociais } \\
\hline Masculino & 32 & 55,99 & \\
\hline Feminino & 30 & 51,11 & \\
\hline Total & 62 & 53,63 & 0,345 \\
\hline \multicolumn{4}{|l|}{ Meio ambiente } \\
\hline Masculino & 32 & 63,67 & \\
\hline Feminino & 30 & 55,84 & \\
\hline Total & 62 & 59,88 & 0,131 \\
\hline \multicolumn{4}{|l|}{ Idade (anos) } \\
\hline \multicolumn{4}{|l|}{ Físico } \\
\hline $18-40$ & 32 & 59,68 & \\
\hline 41 ou mais & 30 & 49,77 & \\
\hline Total & 62 & 54,72 & 0,018 \\
\hline \multicolumn{4}{|l|}{ Psicológico } \\
\hline $18-40$ & 32 & 59,81 & \\
\hline 41 ou mais & 30 & 46,24 & \\
\hline Total & 62 & 53,02 & 0,002 \\
\hline \multicolumn{4}{|l|}{ Relações sociais } \\
\hline $18-40$ & 32 & 61,56 & \\
\hline 41 ou mais & 30 & 45,70 & \\
\hline Total & 62 & 53,63 & 0,001 \\
\hline \multicolumn{4}{|l|}{ Meio ambiente } \\
\hline $18-40$ & 32 & 63,81 & \\
\hline 41 ou mais & 30 & 55,95 & \\
\hline Total & 62 & 59,88 & 0,130 \\
\hline
\end{tabular}

Teste t Student.

Especificamente quanto aos menores escores apresentados neste estudo referentes aos domínios psicológicos e relações sociais, sabe-se que nos últimos anos os acidentes com substâncias radioativas trouxeram com eles graves efeitos psicossociais, que em combinação com outros fatores produziram sérios impactos nas populações atingidas. Essas consequências são bastante complexas pelo fato de não estarem relacionadas somente com o acidente em si, mas aos efeitos de deslocamento e remanejamento das pessoas, assim como ao estigma e à discriminação. Nesse contexto, o acidente ocorrido em Goiânia constituiu-se em um evento bastante negativo, gerando, enquanto evento social, aspectos relacionados à segregação, desorganização e migração. Ao mesmo tempo, enquanto evento psicológico, o choque, o estresse, o medo e o trauma $13,15,19$.

Com relação ao aspecto físico, estudos anteriores a respeito da população de radioacidentados pelo césio-137 em Goiânia demonstraram 

2011.

\begin{tabular}{|c|c|c|c|}
\hline Domínios WHOQOL-BREF & Psicológico & Relações Sociais & Meio Ambiente \\
\hline \multicolumn{4}{|l|}{ Físico } \\
\hline r & 0,738 & 0,712 & 0,736 \\
\hline$p$ & $<0,001$ & $<0,001$ & $<0,001$ \\
\hline \multicolumn{4}{|l|}{ Psicológico } \\
\hline$r$ & - & 0,737 & 0,638 \\
\hline $\mathrm{p}$ & - & $<0,001$ & $<0,001$ \\
\hline \multicolumn{4}{|l|}{ Relações Sociais } \\
\hline r & - & - & 0,617 \\
\hline $\mathrm{p}$ & - & - & $<0,001$ \\
\hline
\end{tabular}

Teste Pearson.

$\mathrm{p}$ : diferem significativamente para $\mathrm{p}<0,001$; r: relação.

que o impacto na saúde física desta população não foi significativo 3,5. É provável que os impactos negativos da exposição à radiação foram evidenciados na percepção dos indivíduos quanto à sua qualidade de vida e permaneceram ao longo do tempo. Segundo Miranda et al. ${ }^{19}$, os problemas de saúde física relatados pelos radioacidentados de Goiânia não se referem diretamente aos efeitos esperados em acidentes dessa natureza, mas atuam de forma indireta entre o aspecto orgânico e o mental, por meio de sintomas somáticos.

Ainda no presente estudo, observou-se que os maiores escores concentraram-se no domínio meio ambiente, talvez porque os aspectos que influenciam uma maior satisfação podem estar relacionados com a salubridade no ambiente físico (clima, ruído, poluição), o que coincide com a maioria das respostas dos sujeitos.

Sabe-se que as estratégias de coping (estilo de enfrentamento) são definidas como um conjunto de esforços, cognitivos e comportamentais, utilizado pelos indivíduos com o objetivo de lidar com demandas específicas, internas ou externas, que surgem em situações de estresse e são avaliadas como sobrecarregando ou excedendo seus recursos pessoais. Nesta pesquisa a variável idade apresentou um considerável índice de significância, mostrando que as pessoas com mais de 41 anos autoavaliaram sua qualidade de vida com escores menores do que as mais jovens. Alguns estudos têm sugerido que os padrões de coping estão associados à idade, ou seja, pessoas mais jovens usam formas de coping mais interativas, tais como a expressão de emoções e a busca de informações, enquanto as mais velhas usam formas mais intrapessoais, como a reflexão e o isolamento social 21,22.

No entanto, apesar do instrumento WHOQOL-BREF requerer uma autoavaliação considerando as duas últimas semanas da data de preenchimento do questionário, deve-se considerar o fato de que na época do acidente radioativo de Goiânia alguns eram crianças e adolescentes. Nesse aspecto, Compas et al. 23 apontam a necessidade de alterações para aplicar as noções de estresse e coping para as crianças, já que precisam ser consideradas a dependência da criança em relação ao adulto e as características básicas do seu desenvolvimento cognitivo e social, pois a idade e as pessoas envolvidas na situação estressante são fatores determinantes na escolha da estratégia a ser utilizada. Geralmente, as estratégias utilizadas por crianças e adolescentes estão mais relacionadas às respostas correspondentes às demandas situacionais, procurando uma adaptação ao ambiente social, indicando que coping é um processo mais situacional do que disposicional, apesar de que não existem respostas adaptativas universais, adequadas para todos os indivíduos, em todas as situações e em todo o tempo 24.

Considerando os aspectos relacionados ao gênero, foi observado neste estudo que a percepção da qualidade de vida para as mulheres foi inferior à dos homens em todas as faixas etárias, nos dois grupos e em todos os domínios, excetuando-se o domínio físico, porém não significativas. Pesquisas têm revelado que as mulheres tendem ao sofrimento psíquico mais do que os homens em situações relacionadas com a saúde e questões sociais, com destaque para aquelas 
que são mães e por isto preocupam-se com os filhos 8,25. A vertente de gênero tem sido identificada como uma questão bastante relacionada com a avaliação da qualidade de vida; especialmente nos casos de eventos traumáticos pode-se verificar que as mulheres geralmente desenvolvem doenças e apresentam prejuízo na saúde mental mais do que os homens 9,25.

Em outro estudo de avaliação da qualidade de vida com pessoas expostas a doses baixas e prolongadas pela radiação ionizante por Cobalto 60 em uma região da cidade de Taiwan, República da China, onde foi utilizado o WHOQOL-BREF, observou-se que os indivíduos mais jovens, as mulheres e as pessoas com maior tempo de exposição apresentaram os menores escores em todos os domínios, com exceção do domínio meio ambiente 26. Esses resultados são semelhantes aos do presente estudo, excetuando-se a variável idade em que as pessoas mais velhas tiveram os escores mais baixos. Talvez isso se deva ao fato de que $o$ acidente de Goiânia foi ocasionado por características bem diferentes em comparação ao de Taiwan, pois aconteceu de forma mais traumática, repentina e impactante e, por sua vez, foi mais presenciado e experimentado pelas pessoas que hoje estão com mais de 41 anos.

Como foi demonstrado, a avaliação dos escores médios dos domínios de qualidade de vida mensurados nesta pesquisa estiveram entre 49,75 e 61,27. Apesar de não terem sido encontrados estudos em populações semelhantes realizados no Brasil, torna-se interessante comparar com trabalhos que utilizaram o instrumento WHOQOL-BREF em populações diversas. Entre eles destaca-se um estudo sobre a Qualidade de Vida e Trauma Psíquico em Vítimas da Violência por Arma de Fogo, cujos resultados demonstraram médias baixas que variaram entre $44,71 \mathrm{e}$ 52,84 , em que o escore médio do domínio meio ambiente foi menor que o dos domínios físico, relações sociais e psicológico 27 .

Outro trabalho que avaliou a Percepção de Qualidade de Vida de Pessoas com Lesão Medular Traumática, apresentou escores entre 49,61 e 55,81, sendo o menor escore representado no domínio meio ambiente e o maior no domínio relações sociais 28 .

Percebe-se nos estudos citados que os menores escores concentraram no domínio meio ambiente, contrariando os resultados da presente pesquisa, cujo escore mais alto foi nesse domínio. Talvez isso se deva aos efeitos comumente chamados "indiretos", os quais podem ser causados por desastres sem ter uma relação direta com o evento, como é o caso dos impactos psicológicos e sociais, sobretudo em eventos com substâncias radioativas, cujas consequências são bastante complexas pelo fato de não estarem relacionadas somente com o acidente em si, mas por preocupações com doenças físicas em longo prazo e pelo impacto decorrente de deslocamentos, segregação, estigma e discriminação 8,19,29.

Nessa perspectiva, uma das principais características do acidente com o césio-137 é que ele não fazia parte da experiência habitual e carregava uma enorme capacidade para gerar pressão física, econômica, social e psicológica sobre os envolvidos. Sendo assim, os resultados demonstraram que as questões preocupantes no início do acidente ainda perduram no presente e no futuro das pessoas afetadas. Essa constatação corrobora com os resultados de outros estudos, confirmando que pelo fato de se tratar de um acidente radioativo, a condição de vítimas permanece, tornando-se crônica 19,20,29

Dentre as limitações deste estudo pode-se destacar a preferência de alguns radioacidentados em se manter na condição de "vítimas", o que pode ter contribuído para uma tendência em uma pior avaliação da qualidade de vida. Isso se justifica, tendo em consideração que os radioacidentados pelo césio-137 de Goiânia sofrem as consequências da violência provocada pelo imprevisível e inesperado, bem como os efeitos prolongados resultantes de acidentes com substâncias radioativas. Geralmente esse efeito se caracteriza pela ameaça crônica e exacerbada por acidentes dessa natureza, gerando necessidades de suporte em áreas de subsistência de suas vidas, tais como: econômica, social, saúde física e mental ${ }^{17}$. Sendo assim, manter a condição de "vítima" pode significar a garantia da sociedade e do governo em gerar respostas a essas necessidades para que as dificuldades sentidas sejam superadas e/ou minimizadas.

Outra limitação foi a dificuldade de convencimento dos radioacidentados, pois, a maioria manifestou revolta com os serviços públicos oferecidos e que não mais serviria de "cobaias" de pesquisa, se negando de forma agressiva a participar do presente estudo. Nesse caso, foi necessário uma articulação com a Associação das Vítimas do Césio-137 (AVCESIO) para ajudar na adesão dos mesmos, até alcançar o quantitativo mínimo do cálculo amostral.

Concluindo, os resultados deste estudo mostraram que os indivíduos expostos à radiação pelo césio-137 sofrem considerável impacto na sua qualidade de vida. Observou-se a persistência de problemas psicossociais, na medida em que, os menores escores foram associados ao domínio psicológico, seguido de relações sociais, sobretudo para os indivíduos do Grupo I, as mulheres e pessoas com mais de 41 anos. Ou seja, enquanto evento psicossocial, esse acidente pode ter pro- 
duzido trauma, medo, estresse, choque, entre outras possíveis reações, até os dias de hoje. Os resultados apontados mostraram que não houve associação ente as variáveis sociodemográficas (religião e escolaridade) com os domínios do WHOQOL-BREF

Contudo, mesmo com a manutenção regular do monitoramento dos indivíduos dos Grupos I e II em Goiânia, verifica-se ainda a existência de incertezas quanto aos efeitos do acidente, pois essas dúvidas desempenham um papel negativo na saúde e no bem-estar dessas pessoas. Esperase com este trabalho que a compreensão sobre a qualidade de vida dos radioacidentados possa subsidiar a elaboração de políticas públicas condizentes e influenciar as decisões e condutas terapêuticas, voltadas a esse segmento da população, incluindo o fortalecimento da atenção psicossocial no intuito de reduzir o sofrimento das mesmas.

Faz-se necessário, também, a realização de novas pesquisas, principalmente porque são muito escassos os estudos que tratam das consequências indiretas em populações de vítimas de acidentes com substâncias radioativas. Enfim, há muito o que se fazer para responder efetivamente às demandas das pessoas que sofrem com os efeitos da radiação ou, pelo menos, minimizar a potencialidade do desenvolvimento de efeitos futuros.

\section{Resumen}

Estudio transversal observacional realizado en Goiânia, Goiás, Brasil, con un 56\% de 111 individuos para evaluar la calidad de vida de las personas expuestas al cesio-137 y su asociación con factores sociodemográficos. Se utilizó el WHOQOL-BREF y el Sistema de Monitoreo de Radio accidentados. Los participantes fueron divididos: Grupo I - Dosimetría radiodermitis y/o citogenética mayor de 20 rads $(n=33)$ y Grupo II dosimetría citogenética $\leq 20 \mathrm{rad}(n=29)$, de un total de 62 sujetos. Entre los dominios del WHOQOL-BREF, el medio ambiente tenía los puntos más altos de promedio $(59,88 ; S D=20,39)$ y el promedio más bajo fue el psicológico $(53,02 ; S D=17,98)$. Las asociaciones entre las relaciones físicas, psicológicas y sociales fueron significativas para la variable edad. No hubo diferencias entre los grupos y entre los factores sociodemográficos y de calidad de vida. Los radioaccidentados sufren un impacto considerable en la calidad de vida, con la persistencia de los problemas psicosociales, especialmente para aquellos con más de 41 años.

Calidad de Vida; Cesio; Impacto Psicosocial; Accidentes

\section{Colaboradores}

S. C. Fuini contribuiu na concepção e design, aquisição de dados e análise e interpretação dos dados, redação do artigo e revisão crítica do conteúdo intelectual. R. Souto fez contribuições substanciais para a concepção e design, análise e interpretação dos dados, e revisão crítica do conteúdo intelectual do artigo. G. F. Amaral e R. G. Amaral fizeram contribuições substanciais para a concepção e design, análise e interpretação dos dados, revisão crítica do conteúdo intelectual e aprovação final da versão a ser publicada.

\section{Agradecimentos}

À equipe do Centro de Assistência aos Radioacidentados (C.A.R.A.), aos colegas do Centro de Ensino, Pesquisa e Projetos - Leide das Neves Ferreira (CEEPP-LNF) e à Associação das Vítimas do Césio-137, pelo auxílio durante a etapa de coleta de dados deste estudo. 


\section{Referências}

1. Oliveira AR, Valverde NJL, Brandão-Mello CE, Almeida CEV. Revisiting the Goiânia accident: medical and dosimetric aspects. Radiat Prot Dosimetry 1998; 77:107-11.

2. International Atomic Energy Agency. The radiological accident in Goiânia. Vienna: International Atomic Energy Agency; 1988.

3. Superintendência Leide das Neves Ferreira. Monitoramento dos radioacidentados. http://www. cesio137goiania.go.gov.br/ (acessado em 20/Dez/ 2009).

4. Goiás. Lei no 17.430 , de 05 de outubro de 2011. Cria as unidades administrativas complementares descentralizadas de saúde que especifica, na Secretaria de Estado da Saúde de Goiás. Diário Oficial do Estado de Goiás 2011; 14 out.

5. Koifman S. Os 20 anos do acidente do césio em Goiás - vigilância ambiental e os riscos para a saúde. http://www.cesio137goiania.go.gov.br/index php?idEditoria=3801 (acessado em 7/Nov/2009).

6. Walker RI, Cerveny RJ. Medical consequences of nuclear warfare. Falls Church: Office of the Surgeon General; 1989.

7. American Psychiatric Association. Diagnostic and stastistical manual of mental disorders. $4^{\text {th }}$ Ed. Washington DC: American Psychiatric Press; 1994.

8. Bromet EJ, Semyon G, Joseph ES, Dmitry G. Somatic symptoms in women 11 years after the Chernobyl accident: prevalence and risk factors. Environ Health Perspect 2002; 110 Suppl 4:625-9.

9. Havenaar J, Rumyantzeva G, Kasyanenko A, Kaasuager K, Westermann N, Brink WVD, et al. Health effects of the Chernobyl disaster: illness or illness behavior: a comparative general health survey in two former Soviet Regions. Environ Health Perspect 1997; 105:1533-7.

10. Fleck MPA. A avaliação da qualidade de vida: guia para profissionais de saúde. Porto Alegre: Editora Artmed; 2008;

11. The WHOQOL Group. Development of the World Health Organization WHOQOL-BREF Quality of life assessment. Psychol Med 1998; 28:551-8.

12. Seidl EMF, Zannon CMLC. Qualidade de vida e saúde: aspectos conceituais e metodológicos. Cad Saúde Pública 2004; 20:580-8.

13. Leon GR. Special issue: overview of the psychosocial impact of disasters. Prehosp Disaster Med 2011 26:1-127.

14. Likh IA, Kovgan LN, Jabob P, Anspaugh LP. Chernobyl accident retrospective and prospective estimates of external dose of the population of Ukraine. Health Phys 2002; 3:290-303.

15. Helou S, Costa Neto SB. Conseqüências psicossociais do acidente de Goiânia. Goiânia: Editora UFG; 1995.

16. Backer P. Gestão ambiental: administração verde. Rio de Janeiro: Qualitymark; 1995.
17. Mattar FN. Pesquisa de marketing. Edição Compacta. 3a Ed. São Paulo: Editora Atlas; 2001.

18. Fleck MPA, Louzada S, Xavier M, Chachamovich E, Vieira G, Santos L, et al. Aplicação da versão em português do instrumento abreviado de avaliação da qualidade de vida "WHOQOL-BREF". Rev Saúde Pública 2000; 34:178-83.

19. Miranda FJ, Pasquali L, Costa Neto SBC, Barreto MQ, David Filho G, Rosa TV. Acidente radioativo de Goiânia: "O tempo cura todos os males?" Arq Bras Psicol 2005; 57:58-87.

20. Rahu M. Health effects; of the Chernobyl accident: fears, rumours and the truth. Eur J Cancer 2000; 39:295-9.

21. Folkman S. Coping across the life span: theoretical issues. In: Cummings EM, Greene AL, Karraker KH, editors. Life span developmental psychology: perspectives on stress and coping. Hillsdale: Lawrence Erlbaum Associates; 1991. p. 3-19.

22. Baum A, Fleming R, Singer JE. Coping with victimization by technological disaster. J Soc Issues 1983; 39:117-38.

23. Compas BE, Banez GA, Malcarne V, Worsham N. Perceived control and coping with stress: a developmental perspective. J Soc Issues 1991; 47:23-34.

24. Dell'Aglio DD, Hutz CS. Estratégias de coping de crianças e adolescentes em eventos estressantes com pares e adultos. Psicologia USP 2002; 13:203-25.

25. Fullerton CS, Ursano RJ. The other side of chaos: understanding the patterns of post-traumatic responses. In: Fullerton CS, Ursano RJ, editors. Posttrumatic stress disorder: acute and long-term responses to trauma and disaster. Washington DC: American Psychiatric Press; 1997. p. 3-18.

26. Pimei NY, Chen-Chang Y, Peter WC, Jing-Shiang H, Hui-Chen L, Kuan-Liang K, et al. Perception of quality of life of a cohort population years after relocation from previous low-dose radiation exposure in Co-60 contaminated buildings in Taiwan. Int J Radiat Biol 2011; 87:453-60.

27. Mello-Silva ACC, Brasil VV, Minamisava R, Oliveira LMAC, Cordeiro JABL, Barbosa MA. Qualidade de vida e trauma psíquico em vítimas da violência por arma de fogo. Texto Contexto-Enferm 2012; 21: 558-65.

28. Bampi LNS, Guilhem D, Lima DD. Qualidade de vida em pessoas com lesão medular traumática: um estudo com o WHOQOL-BREF. Rev Bras Epidemiol 2008; 11:67-77.

29. Loganovsky KN, Loganovoskaya TK. Shizophrenia spectrum disorders in persons exposed to ioniz ing radiation as a result of the Chernobyl accident. Shizophr Bull 2000; 26:751-73.

Recebido em 17/Set/2012

Versão final reapresentada em 08/Fev/2013

Aprovado em 11/Mar/2013 